Military Technical College

Kobry Elkobbah,

Cairo, Egypt

May 29-31,2012

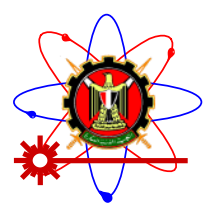

$6^{\text {th }}$ International Conference

on Mathematics and

Engineering Physics

(ICMEP-6)

\title{
NUMERICAL STUDY OF UNSTEADY CIRCULAR PIPE FLOW OF A DUSTY FLUID THROUGH A POROUS MEDIUM
}

\author{
Hazem Ali Attia ${ }^{1}$ Karem Mahmoud Ewis Ibrahim Hamdy Abd Elmaksoud \\ Department of Engineering Mathematics and Physics, \\ Faculty of Engineering, Fayoum University, \\ El-Fayoum-63415, Egypt
}

\begin{abstract}
In this paper, the unsteady flow in a porous medium of a dusty viscous incompressible fluid through a circular pipe is studied taking the Hall effect into consideration. A constant pressure gradient in the axial direction and a uniform magnetic field directed perpendicular to the flow direction are applied. The particle-phase is assumed to behave as a viscous fluid. A numerical solution for the governing equations of motion is developed using the method of finite differences.
\end{abstract}

Keywords: Fluid mechanics, porous medium, computational fluid, flow in channels, circular pipe flow.

\section{Introduction}

The flow of a dusty fluid through a circular pipe has important applications in pumps, accelerators, and flow meters. The performance and efficiency of these devices are influenced by the presence of suspended solid particles in the form of ash or soot as a result of the corrosion and wear activities and/or the combustion processes in accelerators. There have been many articles dealing with theoretical modeling and experimental measurements of the particle-phase viscosity in a dusty fluid (Soo 1969, Gidaspow et al. 1986, Grace 1982, and Sinclair et al. 1989).

The flow of a Newtonian conducting fluid in a circular pipe has been investigated by many authors. Gadiraju et al. (1992) investigated steady two-phase vertical flow in a pipe. Dube et al. (1975) and Ritter et al. (1977) reported solutions for unsteady dusty-gas flow in a circular pipe in the absence of a magnetic field and particle-phase viscous stresses. Chamkha (1994) obtained exact solutions which generalize the results reported

\footnotetext{
${ }^{1}$ ah1113@yahoo.com
} 
Military Technical College

Kobry Elkobbah, Cairo, Egypt

May 29-31,2012

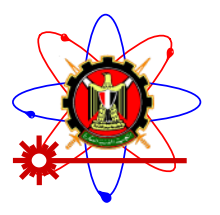

$6^{\text {th }}$ International Conference

on Mathematics and

Engineering Physics

(ICMEP-6)

in Dube et al. 1975 and Ritter et al. 1977 by the inclusion of the magnetic and particlephase viscous effects.

In the present study, the unsteady flow through a porous medium of a dusty viscous incompressible fluid through a circular pipe is investigated. The particle phase is assumed to be incompressible pressureless. The flow in the pipe starts from rest through the application of a constant axial pressure gradient while a uniform magnetic field is applied perpendicular to the flow direction. The flow in the porous medium deals with the analysis in which the differential equation governing the fluid motion is based on the Darcy's law which accounts for the drag exerted by the porous medium (Ingham et al., 2002; Khaled et al., 2003). Closed form solution for the governing momentum equations for both the fluid and particle-phases are obtained. The effect of the porosity of the medium, and the particle-phase viscosity on the velocity of the fluid and particle-phases are investigated.

\section{Notation}

$a$ : pipe radius,

$K$ : Darcy permeability,

$C$ : fluid-phase skin-friction coefficient,

$C_{p}$ : particle-phase skin-friction coefficient,

$\gamma:$ porosity parameter,

$N$ : momentum transfer coefficient,

$P$ : pressure gradient,

$Q$ : fluid-phase volumetric flow rate,

$Q_{p}$ : fluid-phase volumetric flow rate,

$r$ : distance in the radial direction,

$t$ : time,

$V$ : fluid-phase velocity,

$V_{p}$ : particle-phase velocity,

$z$ : axial direction,

$\alpha$ : inverse Stokes number,

$B$ : viscosity ratio,

$\phi:$ particle-phase volume fraction,

$k$ : particle loading,

$\mu$ : fluid-phase viscosity,

$\mu_{p}$ : particle-phase viscosity,

$\rho$ : fluid-phase density, 
Military Technical College

Kobry Elkobbah,

Cairo, Egypt

May 29-31,2012 $6^{\text {th }}$ International Conference

on Mathematics and

Engineering Physics

(ICMEP-6)

$\rho_{p}$ : fluid-phase density,

\section{Governing Equations}

Consider the unsteady, laminar, and axisymmetric horizontal flow of a dusty conducting fluid through an infinitely long circular pipe of radius $a$ pumped by a constant pressure gradient. We assume that both phases behave as viscous fluids and that the volume fraction of suspended particles is finite and constant (Chamkha 1994). The flow is through a porous medium where the Darcy's model is assumed (Ingham et al., 2002; Khaled et al., 2003). The governing momentum equations for both fluid and dust particles phases are, respectively given as

$$
\begin{aligned}
& \rho \frac{\partial V}{\partial t}=-\frac{\partial P}{\partial z}+\frac{\mu}{r} \frac{\partial}{\partial r}\left(r \frac{\partial V}{\partial r}\right)+\frac{\rho_{p} \phi}{1-\phi} N\left(V_{p}-V\right)-\frac{\mu}{K} V \\
& \rho_{p} \frac{\partial V_{p}}{\partial t}=\frac{\mu_{p}}{r} \frac{\partial}{\partial r}\left(r \frac{\partial V_{p}}{\partial r}\right)+\rho_{p} N\left(V-V_{p}\right)
\end{aligned}
$$

where $K$ is the Darcy permeability (Khaled et al., 2003), $\partial P / \partial z$ is the fluid pressure gradient, and $N$ is a momentum transfer coefficient (the reciprocal of the relaxation time, the time needed for the relative velocity between the phases to reduce $e^{-1}$ of its original value (Chamkha 1994). In the present work it is assumed that $\rho, \rho_{p}, \mu_{p}$, and $\varphi$ are all constants where the particle-phase pressure is neglected and the particles are being dragged along with the fluid-phase.

The initial and boundary conditions for the fluid and particle phase are

$V(r, 0)=0, V_{p}(r, 0)=0$,

$$
\frac{\partial V(0, t)}{\partial r}=0, \frac{\partial V_{p}(0, t)}{\partial r}=0, V(a, t)=0, V_{p}(a, t)=0
$$

Introducing the following dimensionless variables and parameters

$\bar{r}=\frac{r}{a}, \bar{t}=\frac{t \mu}{\rho a^{2}}, G_{o}=-\frac{\partial P}{\partial z}, k=\frac{\rho_{p} \phi}{\rho(1-\phi)}, \bar{V}(r, t)=\frac{\mu V(r, t)}{G_{o} a^{2}}, \bar{V}_{p}(r, t)=\frac{\mu V_{p}(r, t)}{G_{o} a^{2}}$,

$\alpha=N d^{2} \rho / \mu$ is the inverse Stoke's number,

$B=\mu_{p} / \mu$ is the viscosity ratio,

$\gamma=a^{2} / K$ is the porosity parameter. 
Military Technical College

Kobry Elkobbah, Cairo, Egypt

May 29-31,2012

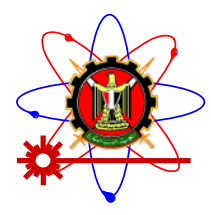

$6^{\text {th }}$ International Conference

on Mathematics and

Engineering Physics

(ICMEP-6)

in Eqs. (1)-(3) we obtain (the bars are dropped for simplicity),

$$
\begin{aligned}
& \frac{\partial V}{\partial t}=1+\frac{\partial^{2} V}{\partial r^{2}}+\frac{1}{r} \frac{\partial V}{\partial r}+k \alpha\left(V_{p}-V\right)-\gamma V \\
& \frac{\partial V_{p}}{\partial t}=B\left(\frac{\partial^{2} V_{p}}{\partial r^{2}}+\frac{1}{r} \frac{\partial V_{p}}{\partial r}\right)+\alpha\left(V-V_{p}\right) \\
& V(r, 0)=0, V_{p}(r, 0)=0, \\
& \frac{\partial V(0, t)}{\partial r}=0, \frac{\partial V_{p}(0, t)}{\partial r}=0, V(1, t)=0, V_{p}(1, t)=0
\end{aligned}
$$

The volumetric flow rates and skin-friction coefficients for both the fluid and particle phases can be defined, respectively, as (Chamkha 1994)

$$
Q=2 \pi \int_{0}^{1} r V(r, t) d r, Q_{p}=2 \pi \int_{0}^{1} r V_{p}(r, t) d r, C=-\frac{\partial V(1, t)}{\partial r}, C_{p}=-B k \frac{\partial V_{p}(1, t)}{\partial r}
$$

\section{Results and Discussion}

Equations (4) and (5) represent a coupled system of partial differential equations which are solved numerically under the initial and boundary conditions (6), using the finite difference approximations. The Crank-Nicolson implicit method (Mitchell et al. 1980 and Evans et al. 2000) is used at two successive time levels. The resulting block tridiagonal system is solved using the generalized Thomas algorithm (Mitchell et al. 1980 and Evans et al. 2000). Computations have been made for $\alpha=1$ and $k=10$. Gridindependence studies show that the computational domain $0<t<\infty$ and $0<r<1$ can be divided into intervals with step sizes $\Delta t=0.0001$ and $\Delta r=0.005$ for time and space respectively. It should be mentioned that the results obtained herein reduce to those reported by Dube et al. (1975) and Chamkha (1994) for the cases of non-magnetic, inviscid particle-phase $(B=0)$, and when neglecting the Hall effect $(m=0)$. These comparisons lend confidence in the accuracy and correctness of the solutions.

Table 1 presents the steady state values of the fluid-phase volumetric flow rate $Q$, the particle-phase volumetric flow rate $Q_{p}$, the fluid-phase skin friction coefficient $C$, and the particle-phase skin friction coefficient $C_{p}$ for various values of the parameters $B$ and $\gamma$. In these computations $\alpha=1$ and $k=10$. It is clear that increasing the parameter $B$ 
Military Technical College

Kobry Elkobbah,

Cairo, Egypt

May 29-31,2012

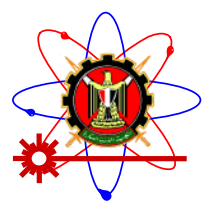

$6^{\text {th }}$ International Conference

on Mathematics and

Engineering Physics

(ICMEP-6)

decreases the quantities $Q, Q_{p}$, and $C$, but increases $C_{p}$ for all values of $\gamma$. This can be explained by the fact that increasing $B$ increases viscosity and therefore the flow rates of both phases as well as the fluid-phase wall friction decreases considerably. However, since $C_{p}$ is defined as directly proportional to $B$, it increases as $B$ increases at all times. Increasing the porosity parameter $\gamma$ decreases the quantities $Q, Q_{p}, C$ and $C_{p}$ for all values of $B$. This is attributed to the fact that the porosity of the medium gives rise to a drag-like or resistive force and it has the tendency to slow down or suppress the movement of the fluid in the pipe, which in turn, reduces the motion of the suspended particle-phase. This is translated into reductions in the average velocities of both the fluid- and the particle-phases and, consequently, in their flow rates. In addition, the reduced motion of the particulate suspension in the pipe as a result of increasing the strength of the magnetic field causes lower velocity gradients at the wall. This has the direct effect of reducing the skin-friction coefficients of both phases.

\section{Conclusion}

The unsteady flow through a porous medium of a particulate suspension in an incompressible viscous fluid in a circular pipe is studied. The governing partial differential equations are solved numerically using the method of finite differences. The effect of the porosity of the medium $\gamma$ and the particle-phase viscosity $B$ on the transient behavior of the velocity, volumetric flow rates, and skin friction coefficients of both fluid and particle-phases is investigates. It is shown that increasing the porosity parameter decreases the fluid and particle velocities.

\section{References}

1. S.L. Soo, "Pipe flow of suspensions", Appl. Sci. Res., vol. 21, pp. 68-84, 1969.

2. D. Gidaspow, "Hydrodynamics of fluidization and heat transfer: super computer modeling", Appl. Mech. Rev., vol. 39, pp. 1-23, 1986.

3. J.R. Grace, Fluidized-Bed Hydrodynamic, Handbook of Multiphase Systems, G. Hetsoroni, Ed., Ch. 8.1, McGraw-Hill, New York. 1982.

4. J.L. Sinclair and R. Jackson, "Gas-particle flow in a vertical pipe with particle-particle interactions", AICHE J., vol. 35, pp. 1473-1486, 1989.

5. M. Gadiraju, J. Peddieson, and S. Munukutla, "Exact solutions for two-phase vertical pipe flow", Mechanics Research Communications, vol. 19, no. 1, pp. 7-13, 1992. 
Military Technical College

Kobry Elkobbah, Cairo, Egypt

May 29-31,2012

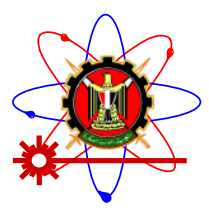

$6^{\text {th }}$ International Conference

on Mathematics and

Engineering Physics

(ICMEP-6)

6. S.N. Dube, and C.L. Sharma, "A note on unsteady flow of a dusty viscous liquid in a circular pipe", J. Phys. Soc. Japan, vol. 38, no. 1, pp. 298-310, 1975.

7. J.M. Ritter, and J. Peddieson, J., "Transient two-phase flows in channels and circular pipes", Proc. 1977 the Sixth Canadian Congress of Applied Mechanics, 1977.

8. A.J. Chamkha, "Unsteady flow of a dusty conducting fluid through a pipe", Mechanics Research Communications, vol. 21, no. 3, pp. 281-286, 1994.

9. A.B. Metzner, "Heat Transfer in non-Newtonian fluid", Adv. Heat Transfer, vol. 2, pp. 357-397, 1965.

10. A. Nakayama, and H. Koyama, "An analysis for friction and heat transfer characteristics of power-law non-Newtonian fluid flows past bodies of arbitrary geometrical configuration", Warme-und Stoffubertragung, vol. 22, pp. 29-37, 1988.

11. H.A. Attia, "Unsteady flow of a dusty conducting non-Newtonian fluid through a pipe", Can. J. Phys., vol. 81, no. 3, pp. 789-795, 2003.

12. H.A. Attia and M.E.S. Ahmed, "Circular pipe MHD flow of a dusty Bingham fluid", In press in Tamkang Journal of Science and Engineering, Tamkang University, Amvo Publishing Company, Taiwan, 2005.

13. G.W. Sutton, and A. Sherman, Engineering Magnetohydrodynamics, McGraw-Hill, New York. 1965.

14. A.R. Mitchell and D.F. Griffiths, The finite difference method in partial differential equations, John Wiley \& Sons, New York. 1980.

15. G.A. Evans, J.M. Blackledge, and P.D. Yardley, Numerical methods for partial differential equations, Springer Verlag, New York. 2000.

Table 1

The steady state values of $Q, Q_{\mathrm{p}}, C, C_{\mathrm{p}}$ for various values of $B$ and $\gamma$

\begin{tabular}{|c|c|c|}
\hline$B=0$ & $\gamma=0.25$ & $\gamma=1$ \\
\hline$Q$ & 0.3032 & 0.2471 \\
\hline$Q_{\mathrm{p}}$ & 0.2582 & 0.1855 \\
\hline$C$ & 0.4125 & 0.3579 \\
\hline$C_{\mathrm{p}}$ & 0 & 0 \\
\hline
\end{tabular}

\begin{tabular}{|c|c|c|}
\hline$B=0.5$ & $\gamma=0.25$ & $\gamma=1$ \\
\hline$Q$ & 0.1764 & 0.1675 \\
\hline$Q_{\mathrm{p}}$ & 0.0426 & 0.0403 \\
\hline$C$ & 0.2818 & 0.2726 \\
\hline$C_{\mathrm{p}}$ & 0.2111 & 0.2003 \\
\hline
\end{tabular}

\begin{tabular}{|c|c|c|}
\hline$B=1$ & $\gamma=0.25$ & $\gamma=1$ \\
\hline$Q$ & 0.1640 & 0.1564 \\
\hline$Q_{\mathrm{p}}$ & 0.0226 & 0.0215 \\
\hline
\end{tabular}


Military Technical College Kobry Elkobbah, Cairo, Egypt May 29-31,2012

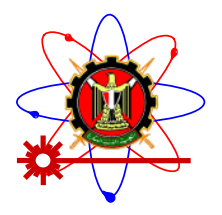

0.2622 $6^{\text {th }}$ International Conference on Mathematics and Engineering Physics (ICMEP-6)

\begin{tabular}{|c|c|c|}
\hline$C$ & 0.2702 & 0.2622 \\
\hline$C_{\mathrm{p}}$ & 0.2231 & 0.2129 \\
\hline
\end{tabular}

